\title{
Design and Evaluation of Modified Adaptive Block Normalized Algorithm for Acoustic Echo Cancellation in Hands-Free Communications
}

\author{
Azeddine Wahbi ${ }^{1 *}$, Ahmed Roukhe ${ }^{2}$ and Laamari Hlou ${ }^{1}$ \\ ${ }^{1}$ Laboratory of Electrical Engineering and Energy System, Faculty of Science, Ibn Tofail \\ University, B.P 133. Kenitra, Morocco \\ ${ }^{2}$ Laboratory of Atomic, Mechanical, Photonics and Energy Faculty of Science, University \\ Moulay Ismail, Meknes, Morocco \\ *corresponding author E-mail: wahbi_azeddine@yahoo.fr
}

\begin{abstract}
The aim of this paper is to design and simulate an AEC in order to enhance the quality of speech disturbed by echo phenomenon. Therefore, in order to design the normalized adaptive AEC, we have used digital signal processing techniques, especially Simulink embedded functions. Effectiveness of the suggested AEC using adaptive normalized algorithm was verified using Matlab/Simulink software. Finally, our AEC has been tested by using ERLE criteria and the analysis results show more efficiency according to ITU-T recommendation G.168.
\end{abstract}

Keywords: Digital Filters, Modified Block Normalized Adaptive Algorithm, Acoustic Echo Cancellation (AEC), Hands-free communication, SimulinklMatlab, Embedded functions, Mean Square Error (MSE), Echo Return Loss Enhancements (ERLE). 


\section{Introduction}

Acoustic Echo Cancellation (AEC) is a technique that significantly enhances the audio quality of a hands-free communications system. Hence, it renders call progress as smooth as possible and offers more comfort to the callers by suppressing or lessening echo phenomenon [1].

Adaptive filters have emerged as an important technology in digital signal processing and real time application, especially acoustic echo cancellation systems. With its ability to remove the echo from original speech, the adaptive filters become a key solution of an AEC.

Different works of adaptive algorithms for echo cancellation are presented [2-3-4]. In [5], we modeled and designed an AEC by using a pre-defined model block adaptive filter to cancel acoustic echo in hands-free communications.

The goal of this work is to design and verify the performance of a software AEC using linear adaptive filter and digital signal processing techniques. In this step, we propose a design of modified block efficient Normalized Adaptive filter based embedded functions. It's used to enhance the quality of speech in spite of echo phenomenon.

The paper is structured as follows: section 2 presents echo cancellation problem formulation, section 3 presents model design and simulation results, and Section 4 concludes this paper.

\section{Echo Cancellation Problem Formulation}

\subsection{Adaptive Filters for modeling AEC}

The outcome of this modeling technique is to help design an AEC that can be carried out by using adaptive filters while keeping in mind that these filters might be Finite Impulse Reponses (FIR) filters or Infinite Impulse Response (IIR) filters.

All the way through this work, we have exclusively implemented FIR filters to achieve the modeling of Acoustic echo cancellers based on normalized adaptive algorithms in time domain. Figs. 1 and 2 respectively illustrate system's diagram block.

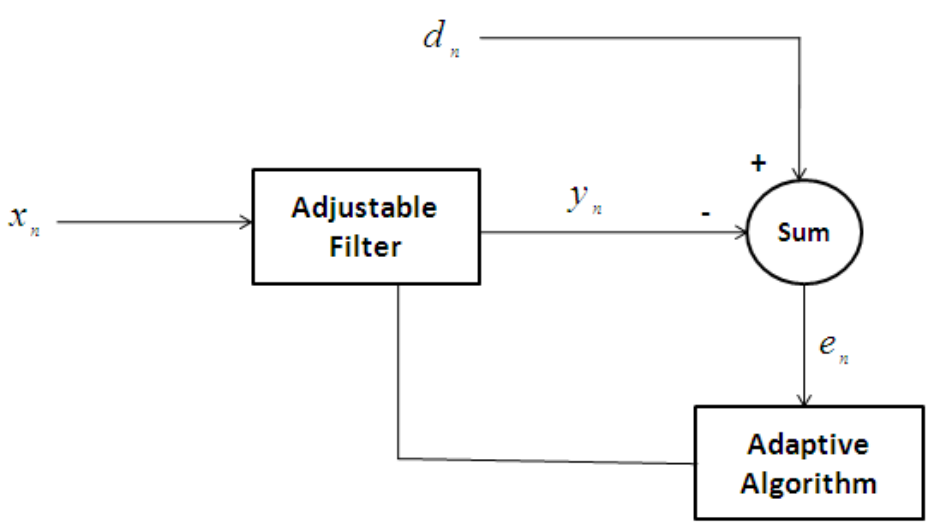

Figure 1. Normalized Adaptative filter structure 


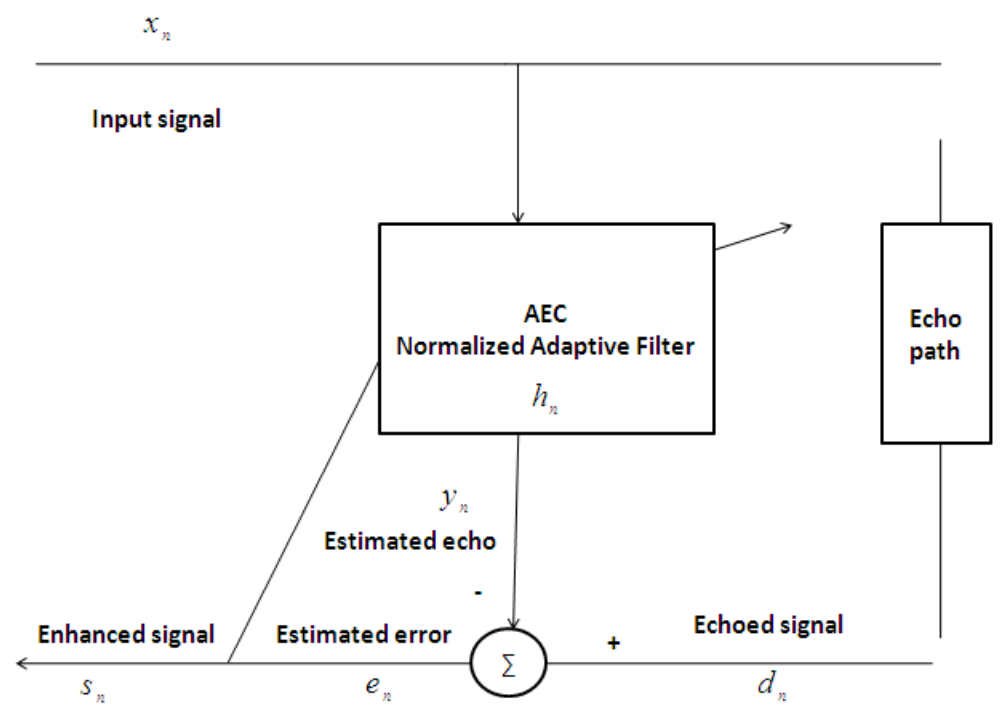

Figure 2. Acoustic echo canceller based on normalized algorithm Block diagram

This algorithm involves two steps:

-estimate the echo signal by using the following equation:

$$
y_{n}=\sum_{k=0}^{N-1} h_{k} x_{n}(k)
$$

Where $h_{n}(k)$ are the filter coefficients and $N$ is the filter length. Therefore $y_{n}$ is the convolution (inner product) of the two vectors $h_{n}$ and $x_{n}$.

-update the of adaptive filter weights by minimizing a cost function (Mean Square Erreur (MSE):

$$
M S E=\frac{1}{2} \sum_{k}\left(e_{n}\right)^{2}
$$

Where,

$$
\begin{array}{r}
e_{n}=d_{n}-y_{n} \\
h_{n}=h_{n+1}+\frac{\lambda}{\beta+\left\|x_{n}\right\|^{2}} x_{n} e_{n}
\end{array}
$$

\subsection{Modified N LMS Block Algorithm}

In this subsection, we designed a modified block in the order to evaluate the complexity of the echo path by means of a normalized adaptive algorithm. This Block may be designed 
by using digital signal processing techniques and embedded functions in Matlab Simulink environment [6].

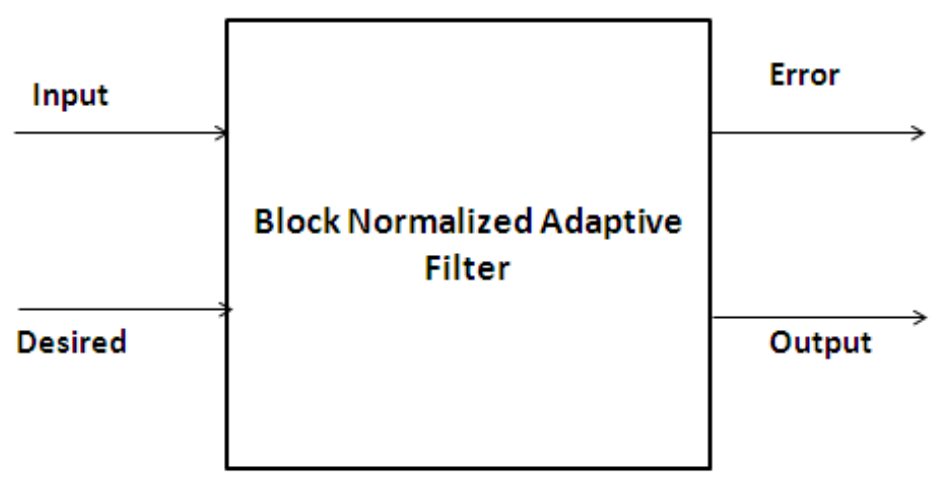

Figure 3. Modified NLMS filter block.

The block estimates the filter weights $h_{n}$ needed to minimize the error, between output $y_{n}$ and $d_{n}$.

Where $y_{n}$ and $d_{n}$ respectively designate the estimated echo and echoed signal, while $e_{n}$ represent the estimated error.

Widrow \& al [7] formulated the LMS algorithm for obtaining the minimum output power.

On the other hand, a periodic echoed signal has a very long correlation time [8].

Hence, we define the primary input signal to be a delayed version of the measurement $\mathrm{y}_{\mathrm{n}}$ and the reference signal to be the measurement itself.

We then apply the following modified normalized algorithm which tracks the desired solution.

The variables of normalized filter can be summarized as follows.

Table 1. Variable of normalized filter

\begin{tabular}{|l|l|}
\hline \multicolumn{1}{|c|}{ Variable } & \multicolumn{1}{c|}{ Description } \\
\hline \hline$n$ & the present step of the algorithm \\
\hline$x_{n}$ & the input signal at step $n$ \\
\hline$h_{n}$ & The adaptive filter coefficients at step $n$ \\
\hline$y_{n}$ & the estimated echo at step $n$ \\
\hline$d_{n}$ & the echoed signal at step $n$ \\
\hline$e_{n}$ & the estimated error at step $n$ \\
\hline$s_{n}$ & the enhanced signal at step $n$ \\
\hline$\lambda$ & the step to adjust. where $0<\lambda<1$ \\
\hline$\beta$ & small number inserted in the denominator to avoid division by zero. \\
\hline
\end{tabular}


Summary Flowchart in Fig. 4 describes the flow of the adaptive algorithm in time domain.

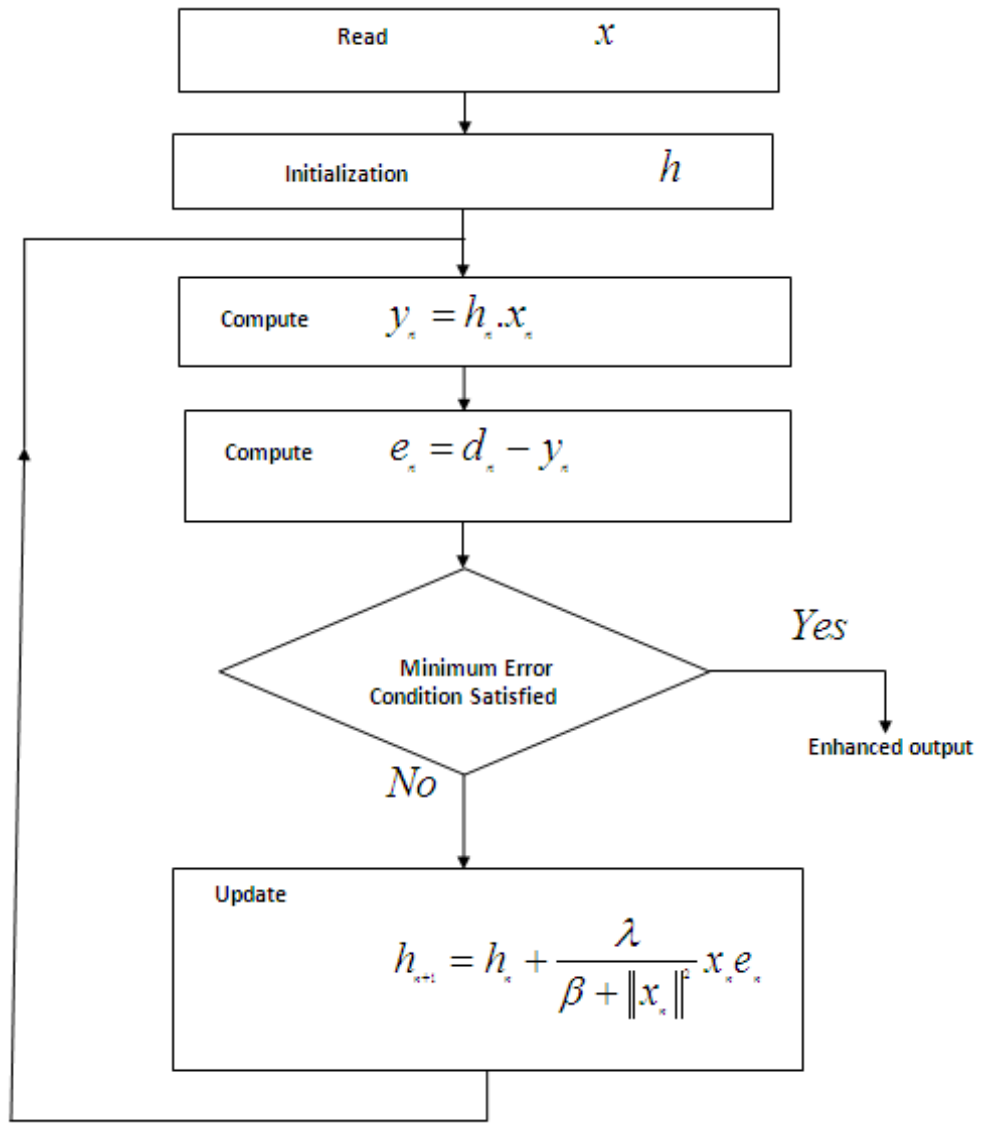

Figure 4. Flowchart of normalized adaptive algorithm

\section{Model design and Simulink Results}

\subsection{Echo Canceller design using Matlab/Simulink}

The overall performance of the module is guaranteed as shown in Fig. 5. It is designed and verified by using the following parameters:

The delay time is 32 (ms) and the number of samples for each read from file length (From Wave File) is 64 samples per frame at $8000 \mathrm{~Hz}$ sampling rate.

AEC implementation is setup with NLMS adaptive filter having a length of 64 . The step size is chosen as $\lambda=0.00001$. 
Simulation Design of AEC using Normalized Algorithm

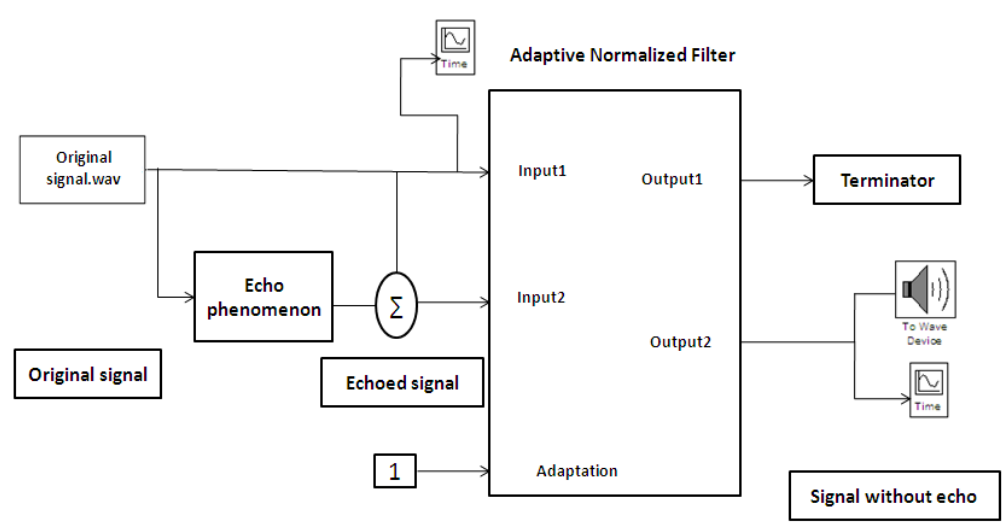

Figure 5. Echo cancellation under Simulink using functional programming

\subsection{Simulink Results}

The AEC modeled throughout this work was tested using Matlab/Simulink software. The original signal and echoed signal used in this simulation are respectively reported by Figs. 6 and 7.

Fig. 8 shows system's output and reveals that speech quality has been improved.

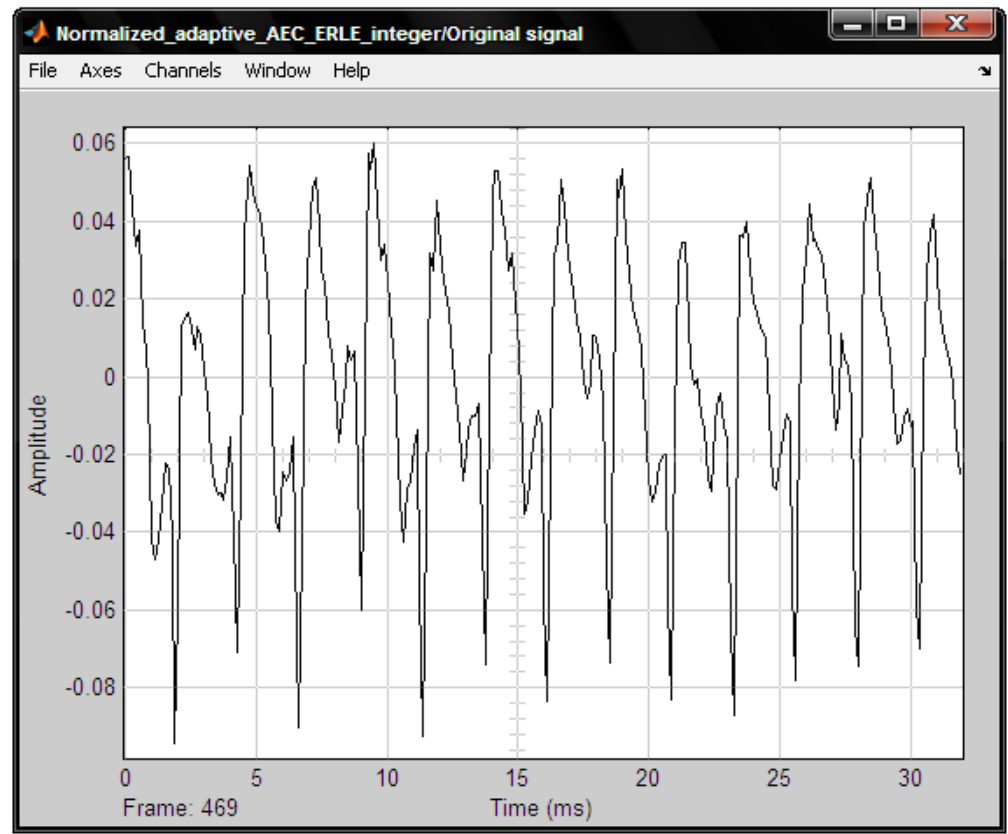

Figure.6. Original signal (Simulink) 


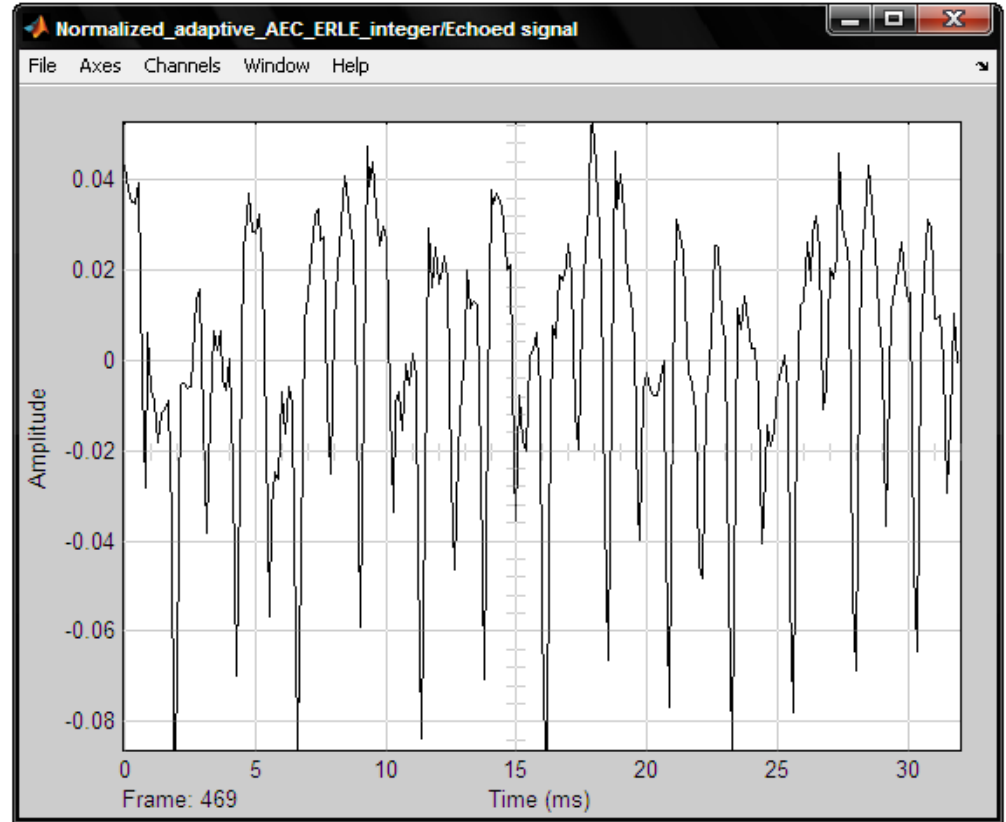

Figure.7. Echoed signal (Simulink)

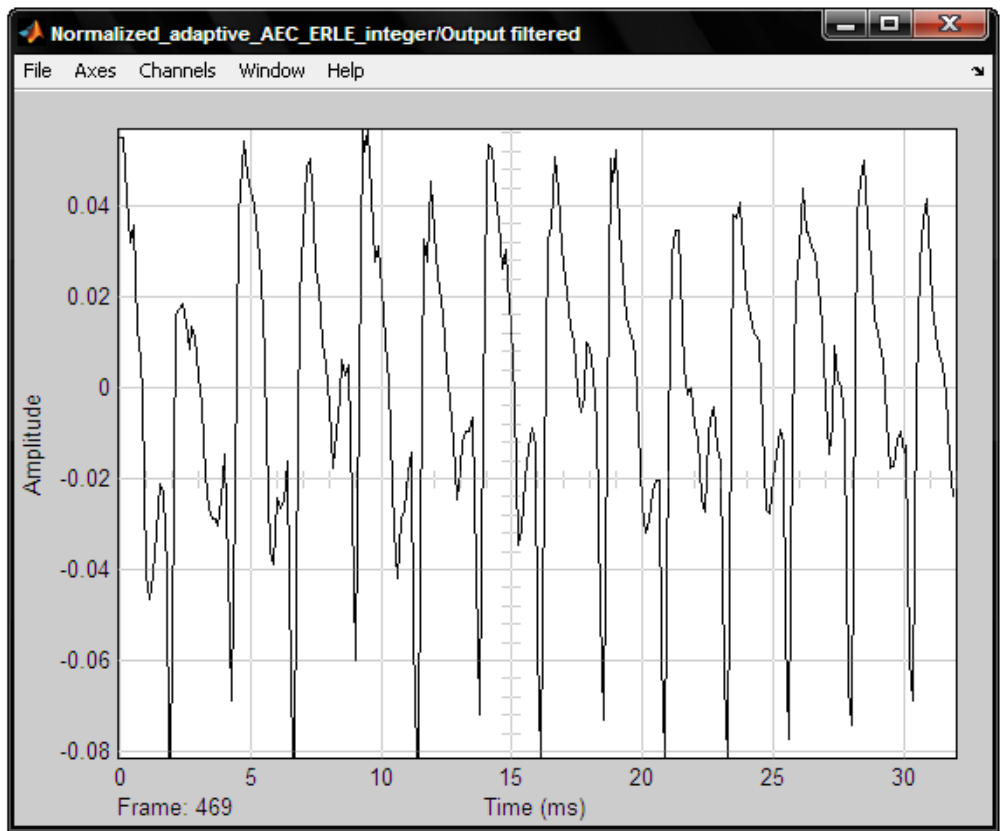

Figure.8. Filtered output (Simulink result)

At this point, we expanded several scenarios to test the convergence of our AEC based on modified normalized adaptive algorithm. For each scenario, we did verify the convergence performance of the algorithm by adjusting step-size and filter length parameters and observed which step-size best values lead to optimal weights. Therefore, the designed AEC provides significant enhancement in the transmission path by reducing the strength of the echoed signal. 


\section{3 performance of the proposed AEC}

The effectiveness of the proposed AEC is verified by using Echo Return Loss Enhancements (ERLE) criteria. It is calculated as:

$$
E R L E_{d B}=10 \log _{10}\left(\frac{\text { Power }(\text { Echoed signal })}{\text { Power }(\text { Output AEC })}\right.
$$

The proposed AEC based Normalized Least Square algorithm has shown an improved ERLE performance according to ITU-T recommendation G.168 [11]. In this context, the simulation results for ERLE are presented in Table 2.

Table 2. ERLE improvement of the proposed AEC $(\mathrm{N}=256, \mathrm{~L}=64, \lambda=0.00001)$

\begin{tabular}{|c|c|}
\hline Time (s) & ERLE of enhanced output (dB) \\
\hline \hline 3 & 27.23 \\
\hline 6 & 27.6 \\
\hline 9 & 20.12 \\
\hline 12 & 20.29 \\
\hline 15 & 29.63 \\
\hline 18 & 18.72 \\
\hline 21 & 22.74 \\
\hline 24 & 14.75 \\
\hline 27 & 28.96 \\
\hline 30 & 27.7 \\
\hline
\end{tabular}

\section{Conclusion}

This paper proposed an AEC based on normalized adaptive algorithm and digital signal processing. With quantifiable results, this work seems to be an efficient method to be used to improve the quality of speech in hands-free communication.

The keypoint of this approach is to design an AEC module for both simulation and real time implementation, taking benefit from the simplicity of programming using functional simulink. The experimental results show that the ERLE values are very reasonable and in accordance with ITU-T recommendation G.168.

Finally, knowing that the rapid development of hands-free communication systems tends to generate a lot of nonlinear acoustical echo, we will therefore design and implement, in a future work, a nonlinear AEC to reject this disturbance. Additionally, we will mainly focus on the improvement and effectiveness of this nonlinear AEC. 


\section{References}

[1] F. Ykhlef et al, “Acoustic Echo Cancellation and Suppression of Noise for hands-free communications”, 5th International Conference: Sciences of Electronic, Technologies of Information and Telecommunication in Tunisia, March 22-26, 2009.

[2] J. Velazquez Lopez, Juan Carlos Sanchez and Hector Perez Meana, “Adaptive Echo Canceller Using a Modified LMS Algorithm”, 2nd International Conference on Electrical and Electronics Engineering (ICEEE) and XI Conference on Electrical Engineering, September 7-9, 2005.

[3] S. Dhamija and Akash Tayal, “Adaptive filtering algorithms for channel equalization and echo cancellation”, International Journal of Computer Technology and Applications, Vol. 2, No. 5, pp. 1577-1581, Sept-Oct 2011

[4] Abdelghani Manseur, Daoud Berkani and Abdenour Mekhmoukh, “Adaptive filtering using Higher Order Statistics (HOS)”, International Journal of Computer Science, Vol. 9, Issue 2, No 1, March 2012.

[5] Wahbi Azeddine, Rachid El Gouri, Anas El Habti El Idrissi, Hlou Laamari, "Real-time acoustic echo cancelation for hands-free communications by implementing a LMS algorithm onboard an autonomous DSK C6713”, 3rd International Conference on Systems, Modeling and Design (MIC-SMD 2013): 1-3 February 2013, Kenitra, Morocco.

[6] The Mathworks Inc., Matlab and Simulink User's Guide, 2012.

[7] Widrow, B., Glover, J.R., Jr. ; McCool, J.M. ; Kaunitz, J. ; Williams, C.S. ; Hearn, R.H. ; Zeidler, J.R. ; Eugene Dong, Jr. ; Goodlin, R.C., “Adaptive Noise Cancelling: Principals and Applications” Proceeding of the IEEE, vol. 63, No. 12, pp.1692-1716, Dec. 1975.

http://dx.doi.org/10.1109/ACSSC.2009.5470150.

[8] I. Homana, M.D. Topa, B.S. Kirei, “Echo cancelling using adaptive algorithms”, 15th International Symposium for Design and Technology of Electronics Packages, pp. 317-321, Sept.2009. http://dx.doi.org/ 10.1109/SIITME.2009.5407351

[9] C.Paleologu, J.Benesty, S.L.Grant, C.Osterwise, "Variable step-size NLMS algorithms for echo cancellation”, Conference Record of the forty-third Asilomar Conference on Signals, Systems and Computers, pp. 633-637, Nov 2009. http://dx.doi.org/10.1109/ACSSC.2009.5469916 [10] Radhika Chinaboina, D.S.Ramkiran, Habibulla Khan, M. Usha, B.T.P.Madhav, K.Phani Srinivas, \& G.V.Ganesh, ' Adaptive algorithms for acoustic echo cancellation in speech processing”, International Journal of Research and Reviews in Applied Sciences, Vol. 7, No. 1, April 2011.

[11] UIT-T, Recommendation G.168, "Digital network echo cancellers", June 2002.

\section{Copyright Disclaimer}

Copyright reserved by the author(s).

This article is an open-access article distributed under the terms and conditions of the Creative Commons Attribution license (http://creativecommons.org/licenses/by/3.0/). 